\title{
Consistency of the Periodogram When the Long-Run Variance is Degenerate
}

\author{
Jin Lee ${ }^{1, a}$ \\ ${ }^{a}$ Department of Economics, Ewha Womans University
}

\begin{abstract}
Sample periodogram is widely known as an inconsistent estimator for true spectral density. We show that it becomes consistent when the true spectrum at the zero frequency (often known as long-run variance) equals zero. Asymptotic results for consistency of the periodogram as well as the rate of convergence are formally derived.
\end{abstract}

Keywords: Periodogram, spectrum, long-run variance, consistency.

\section{Introduction}

In an economics or econometrics context, the power spectrum at the zero frequency draws the most attention, as the magnitude of spectrum at the origin is interpreted as long-run information contained in the data. Spectral density at the origin (ignoring the constant term) equals to infinite sum of covariances of underlying processes, where covariance reflects the relationship between the current and past information or between the two stationary variables (Andrews, 1991). In that sense, the sum of all the variance and covariances is often called long-run variance that reflects long-run information in the time series data (Granger, 1969; Hamilton, 1994; Phillips, 2005). Meanwhile, estimation for the zero-frequency spectrum or long-run variance estimation has been an important task. Long-run variance arises in the presence of possible heteroskedasticity and autocorrelation of unknown forms in diverse econometric models such as linear, nonlinear, GMM and cointegrating estimation. As a robust estimator to heteroskedasticity and autocorrelation, kernel-based nonparametric estimators have been widely used and is commonly referred to HAC(heteroskedasticity and autocorrelation consistent) estimator in time series literature (Andrews, 1991; Newey and West, 1994). When the true spectrum at the origin is assumed to be strictly positive, kernel weighted estimators, equipped with the bandwidths, achieve consistency (Priestley, 1981).

Periodogram as a spectral density estimator at the origin is defined as unweighted sums of sample covariances of stationary time series process. Applications of using periodogram have been wide. Business cycle estimation (among them) remains a popular example of economic application using periodogram techniques. Estimated power spectrum based on periodogram estimates indicates information on business cycles of different frequencies including long-term, mid-term trends and seasonal effects (Hamilton, 1994). Periodogram has been widely used to analyze long memory time series. Given the spectral behavior of long-range dependent process, one can use periodogram estimates at the zero frequency to estimate the long memory parameter (Brockwell and Davis, 1990). Though an asymptotically unbiased estimator, periodogram is not a consistent estimator for the true spectrum at any frequency. Inconsistency results follow from the fact that the asymptotic variance of periodogram

\footnotetext{
${ }_{1}^{1}$ Associate Professor, Department of Economics, Ewha Womans University, Daehyun-Dong, Seodaemun-Gu, Seoul 120-

750, Korea. E-mail: leejin@ewha.ac.kr
} 
does not decay to zero with the sample size, which is a well known property in the spectral time series context. For theoretical properties of periodogram, see Priestley (1981) among others.

In this paper, we focus on a degenerate case where the true spectrum at the origin equals to zero. Degeneracy arises when we mistakenly over-difference the data, which is rendered to be moving average unit root process. For instance, the first-differenced form of a process whose true statistical order of integration is zero generates a process with the integration of order for a negative one or moving average unit root process. The sum of all the variance and covariances of moving average unit root process becomes zero that corresponds to zero spectrum at the origin. Given this degenerate situation, we explicitly show that periodogram can be a consistent estimator. Consistency results can be only obtained in the presence of degeneracy that is otherwise inconsistent in a non-degenerating situation. Thus, as a possible research area, we can consider testing whether the series is over-differenced or not through use of a periodogram see Saikkonen and Lukkonen (1993) and Lee (2010).

\section{Main Results}

We analyze the consistency of periodogram under the null hypothesis that the true long-run variance becomes zero. The periodogram is given as

$$
I(\lambda)=\sum_{j=1-N}^{N-1} \hat{R}(j) \cos (j \lambda), \quad \text { for }-\pi \leq \lambda \leq \pi,
$$

where $\hat{R}(j)=1 / N \sum_{t=|j|+1}^{N}\left(X_{t}-\bar{X}\right)\left(X_{t-j}-\bar{X}\right)$, and $\bar{X}=N^{-1} \sum_{t=1}^{N} X_{t}$.

Define a power spectrum

$$
f(\lambda)=(2 \pi)^{-1} \sum_{j=-\infty}^{\infty} R(j) \cos (j \lambda),
$$

where $-\pi \leq \lambda \leq \pi$ (Priestley, 1981). It is well known that the periodogram is not a consistent estimator in the sense that the variance of the periodogram does not decay to zero with the sample size or under certain conditions, $\operatorname{Var}(I(\lambda))=O(1)$.

We restrict our attention to the quantity of the spectrum at the zero frequency, as it contains longrun information in the data. It is often referred to a long-run variance of $\left\{X_{t}\right\}$ in the econometrics context given as

$$
f(0)=(2 \pi)^{-1} \sum_{j=-\infty}^{\infty} R(j) .
$$

As an estimator for (2.2), periodogram at the zero frequency $I(0)$ does not serve as a consistent estimator. Inconsistency, however, is present under the non-degenerating case that the true spectrum $f(0)>0$.

To see this formally, we rewrite the periodogram at the zero frequency $I(0)$ as

$$
I(0)=(2 \pi)^{-1} \sum_{j=1-N}^{N-1} k\left(\frac{j}{N}\right) \hat{R}(j),
$$

where $k(x)=1$ for $|x| \leq 1$, and $=0$ otherwise. The weighting function $k$ is a special form of kernel function with the bandwidth equal to the sample size. Intuitively, one cannot obtain a consistency 
result when the bandwidth equals to the sample size. Our work shows one important exception caused by the degenerate situation.

In order to prove the consistency, first, we need some conditions for the data generating processes and for spectral density function $f(\lambda)$ at $\lambda=0$.

\section{Assumption 1.}

(a) $X_{t}$, for $t=1,2, \ldots, N$ is covariance stationary time series process.

(b) $\sum_{j=-\infty}^{\infty}|R(j)|<\infty$, and $\sum_{j=-\infty}^{\infty}|j|^{q}|R(j)|<\infty$, for $q \in[0, \infty)$, where $R(j)=E\left(X_{t}-\mu\right)\left(X_{t-j}-\mu\right)$, and $\mu=E\left(X_{t}\right)$.

The smoothness condition in part (b) is required for a higher-order Taylor expansion of the function $f(\cdot)$ near zero frequency. Moreover, define the $q^{\text {th }}$ order generalized spectral derivative,

$$
f^{(q)}(0)=(2 \pi)^{-1} \sum_{j=-\infty}^{\infty}|j|^{q} R(j), \quad \text { for } q \in[0, \infty)
$$

where $q$ may not be integer-valued. The generalized spectral derivative $f^{(q)}(0)$ is not necessarily equal to $q^{\text {th }}$ derivative, $d^{q} f(\lambda) /\left.d \lambda^{q}\right|_{\lambda=0}$. If $q$ is even-numbered, then

$$
f^{(q)}(0)=\left.(-1)^{\frac{q}{2}} \frac{d^{q} f(\lambda)}{d \lambda^{q}}\right|_{\lambda=0} .
$$

The larger values of $q$, the more smooth the spectral density function near the origin. When $f(0)=0$, it is necessary to derive higher-order expansions of the estimator near the zero frequency that requires a large values of $q$. Our main results need $q \geq 4$.

Now, we present the main result.

Theorem 1. Suppose Assumptions 1 hold, Under $f(0)=0$,

$$
\lim _{N \rightarrow \infty} N^{4} \operatorname{Var}(I(0))=\Omega,
$$

where $\Omega=\left(4 \pi^{3}\right)\left[f^{(2)}(0)\right]^{2} \int_{-\infty}^{\infty} u^{4} K^{2}(u) d u$ and $K$ is a spectral window of a kernel function.

The results show the consistency of the periodogram when the true spectrum is zero at the origin. Unlike non-degenerating case, the asymptotic variance decays to zero at the rate of $N^{4}$. The proof is in the appendix. As in (2.4), we concentrate on a periodogram with a truncated kernel, i.e., $k(z)=1$, for $z \in[-1,1]$. The associated spectral window is given as

$$
K(\lambda)=\frac{1}{\pi} \frac{\sin (\lambda)}{\lambda} .
$$

As is known in the literature, existing asymptotic results show that the variance of periodogram does not vanish with the sample size in the non-degenerate case. Fundamentally, different results arise in the degenerate case. As in the proof, the idea to derive the consistency is simple. Conventional firstorder asymptotics lead to inclusion of $f(0)$ in the asymptotic variance of the periodogram. Thus, it is necessary to tackle with a higher-order Taylor expansion of the asymptotic variance, and as a result, convergence rates in Theorem 1 have been found. For reference, the consistency of kernel estimators in the degenerate case is studied in Lee (2010). The proof of Theorem 1 is in the Appendix. 


\section{Conclusion}

We show the consistency of a sample periodogram estimator when the true long-run variance is zero at the origin. Asymptotic variance of the periodogram is formally shown to decay with the sample size. Proposed results are different from the existing results of the inconsistency in sample periodogram estimation.

\section{Appendix:}

Proof: (Proof of Theorem 1) We explicitly derive the limit of $\operatorname{Var}(I(0))$ when $f(0)=0$. We make use of similar techniques in Lee (2010). Under Gaussian assumption, we have (see Priestley, 1981)

$$
\operatorname{Var}(I(0))=\sum_{j=1-N}^{N-1} \sum_{j^{\prime}=1-N}^{N-1} k\left(\frac{j}{N}\right) k\left(\frac{j^{\prime}}{N}\right) \operatorname{Cov}\left[\hat{R}(j), \hat{R}\left(j^{\prime}\right)\right],
$$

where

$$
\operatorname{Cov}\left[\hat{R}(j), \hat{R}\left(j^{\prime}\right)\right]=N^{-1} \sum_{h=1-N}^{N-1}\left[R(h) R\left(h+j^{\prime}-j\right)+R\left(h+j^{\prime}\right) R(h-j)(1+o(1))\right] .
$$

Then, we can decompose

$$
\operatorname{Var}(I(0))=\left(V_{1 N}+V_{2 N}\right)(1+o(1))
$$

where

$$
\begin{aligned}
& V_{1 N}=N^{-1} \sum_{j=1-N}^{N-1} \sum_{j^{\prime}=1-N}^{N-1} k\left(\frac{j}{N}\right) k\left(\frac{j^{\prime}}{N}\right) \sum_{h=1-N}^{N-1} R(h) R\left(h+j^{\prime}-j\right), \\
& V_{2 N}=N^{-1} \sum_{j=1-N}^{N-1} \sum_{j^{\prime}=1-N}^{N-1} k\left(\frac{j}{N}\right) k\left(\frac{j^{\prime}}{N}\right) \sum_{h=1-N}^{N-1} R\left(h+j^{\prime}\right) R(h-j) .
\end{aligned}
$$

By using Fourier and inverse Fourier transformations, the $V_{1 N}$ can be written as

$$
\begin{aligned}
N \times V_{1 N}= & \sum_{h=1-N}^{N-1} R(h) \sum_{j=1-N}^{N-1} k\left(\frac{j}{N}\right) \sum_{j^{\prime}=1-N}^{N-1} \int_{-\pi}^{\pi} N K(N \lambda) e^{-i j^{\prime} \lambda} R\left(h+j^{\prime}-j\right) d \lambda \\
= & (2 \pi)^{3}\left[(2 \pi)^{-1} \sum_{h=1-N}^{N-1} R(h)\right]\left[(2 \pi)^{-1} \sum_{j=1-N}^{N-1} k\left(\frac{j}{N}\right)\right] \\
& \times\left[\int_{-\pi}^{\pi} N K(N \lambda)(2 \pi)^{-1} \sum_{j^{\prime}=1-N}^{N-1} R\left(h+j^{\prime}-j\right) e^{-i\left(h+j^{\prime}-j\right) \lambda} e^{i(h-j) \lambda} d \lambda\right]
\end{aligned}
$$

which can be further rearranged as

$$
\begin{aligned}
N \times V_{1 N} & =(2 \pi)^{3}\left[(2 \pi)^{-1} \sum_{h=1-N}^{N-1} R(h)\right]\left[(2 \pi)^{-1} \sum_{j=1-N}^{N-1} k\left(\frac{j}{N}\right)\right]\left[\int_{-\pi}^{\pi} N K(N \lambda) f(\lambda) e^{i(h-j)} d \lambda\right] \\
& =(2 \pi)^{3} \int_{-\pi}^{\pi} N K(N \lambda) f(\lambda)\left[f(\lambda)-(2 \pi)^{-1} \sum_{h \geq N} R(h) e^{i h \lambda}\right][N K(N \lambda)] d \lambda\left(1+o_{p}(1)\right) \\
& =\left(A_{1 N}-B_{1 N}\right)\left(1+o_{p}(1)\right),
\end{aligned}
$$


where

$$
\begin{aligned}
& A_{1 N}=(2 \pi)^{3} \int_{-\pi}^{\pi} f^{2}(\lambda) N^{2} K^{2}(N \lambda) d \lambda, \\
& B_{1 N}=(2 \pi)^{3} \int_{-\pi}^{\pi} f(\lambda) N^{2} K^{2}(N \lambda) \sum_{|h| \geq N} R(h) e^{i h \lambda} .
\end{aligned}
$$

In (A.3), the spectral window of a kernel function is defined as

$$
K(\lambda)=(2 \pi)^{-1} \int_{-\infty}^{\infty} k(x) e^{-i x \lambda} d x, \quad N K(N \lambda)=(2 \pi)^{-1} \int_{-\infty}^{\infty} k\left(\frac{x}{N}\right) e^{-i x \lambda} d x,
$$

(by the change of variables, e.g., Priestley (1981, p.447)).

Now, as for $A_{1 N}$, we obtain

$$
\begin{aligned}
A_{1 N} & =(2 \pi)^{3} \int_{-\pi}^{\pi} f^{2}(\lambda) N^{2} K^{2}(N \lambda) d \lambda \\
& =(2 \pi)^{3} N \int_{-\pi}^{\pi} f^{2}\left(\frac{u}{N}\right) K^{2}(u) d u \\
& =(2 \pi)^{3} N\left[\left(\frac{1}{4}\right) f_{(2)}^{2}(0) \int_{-\pi}^{\pi}\left(\frac{u}{N}\right)^{4} K^{2}(u) d u+O\left(M^{-6}\right)\right] \\
& =2 \pi^{3} N^{-3} f_{(2)}^{2}(0) \int_{-\pi}^{\pi} u^{4} K^{2}(u) d u+O\left(M^{-5}\right),
\end{aligned}
$$

where the second line follows from the change of variable techniques, and the third line from Taylor expansions of the squared $f(0)$.

By similar reasoning, for $B_{1 N}$, we have

$$
\begin{aligned}
B_{1 N} & \leq(2 \pi)^{3} \sum_{|h| \geq N} R(h) \int_{-\pi}^{\pi} f(\lambda) N^{2} K^{2}(N \lambda) d \lambda \\
& \leq(2 \pi)^{3} N^{-q} \sum_{|h| \geq N}|h|^{q} R(h) \int_{-\pi}^{\pi} f(\lambda) N^{2} K^{2}(N \lambda) d \lambda .
\end{aligned}
$$

Again, using Taylor expansions, the integrand term is analyzed as

$$
\begin{aligned}
\int_{-\pi}^{\pi} f(\lambda) N^{2} K^{2}(N \lambda) d \lambda & =N \int_{-\pi}^{\pi} f\left(\frac{u}{N}\right) K^{2}(u) d u \\
& =N\left[\left(\frac{1}{2}\right) f_{(2)}(0) \int_{-\pi}^{\pi}\left(\frac{u}{N}\right)^{2} K^{2}(u) d u\right]+O\left(M^{-4}\right) .
\end{aligned}
$$

It follows that $\int_{-\pi}^{\pi} f(\lambda) N^{2} K^{2}(N \lambda) d \lambda=O\left(N^{-1}\right)$ and as a result,

$$
B_{1 N}=O\left(N^{-q} N^{-1}\right)=O\left(N^{-(1+q)}\right),
$$

which is $o\left(N^{3}\right)$, if $q>2$. 
Similar proofs are applied to $V_{2 N}$ term in (A.2), where the dominating term equals to $A_{1 N}$. We skip this part to save a space. Therefore, we have

$$
N^{4}\left[V_{1 N}+V_{2 N}\right]=\left(4 \pi^{3}\right) f_{(2)}^{2}(0) \int_{-\pi}^{\pi} u^{4} K^{2}(u) d u+o(1)
$$

which implies the consistency result,

$$
\lim _{N \rightarrow \infty} \operatorname{Var}(I(0))=O\left(N^{-4}\right)=o(1) .
$$

Proof of the Theorem 1 is completed.

\section{Acknowledgements}

The author is very grateful to constructive comments by an anonymous referee.

\section{References}

Andrews, D. W. K. (1991). Heteroskedasticity and autocorrelation consistent covariance matrix estimation, Econometrica, 59, 817-858.

Brockwell, P. and Davis, R. (1990). Time Series: Theory and Methods, Springer series in statistics.

Granger, C. (1969). Investigating causal relations by econometric models and cross-spectral methods, Econometrica, 37, 315-340.

Hamilton, J. (1994). Time Series Analysis, Princeton.

Lee, J. (2010). Long-run variance estimation for linear processes under possible degeneracy, Journal of Economic Theory and Econometrics, 21, 1-22.

Newey, W. and West, K. (1994). Automatic lag selection in covariance matrix estimation, Review of Economic Studies, 61, 631-653.

Phillips, P. C. B. (2005). HAC estimation by automated regression, Econometric Theory, 21, 116-142. Priestley, M. B. (1981). Spectral Analysis and Time Series, Academic Press New York.

Saikkonen and Lukkonen (1993). Testing for moving average unit root in autoregressive integrated moving average unit root, Journal of the American Statistical Association, 88, 596-601. 